Research Article

\title{
Automated Teller Machine Network Inspection under Stochastic Modelling
}

\author{
Mangey Ram* and Nupur Goyal \\ Department of Mathematics, Graphic Era University, Dehradun, Uttarakhand-248002, India
}

Received 23 August 2015; Accepted 10 September 2016

\begin{abstract}
Increasingly development in the field of banking investigates new technologies for providing the best services between banks and customers with good efficiency, and encouraged the accommodation of technology providers. Automatic Teller Machine (ATM) has a significant issue in retentive customers as well as achieving an economical advantage although growing and preserving the overall profitability of the channel. ATM technology is developing at frightening rate and growing in industries with exponential rate. The safety and economic of ATM has been most significant concern in networking with the emergence of internetworking technology. The authors investigate the reliability measures of a repairable ATM system, by which reliability engineers or designers can determine how reliability can be improved using appropriate vicissitudes and also seek the sensitivity analysis for several variations in reliability characteristics along with the modifications in precise values of the input parameters.
\end{abstract}

Keywords: Complex Banking System; Automatic Teller Machine (ATM); Stochastic modelling; Reliability measures.

\section{Introduction}

In the worldwide, ATM network is very significant in human life. It has been developed excessively during the last two decades and delivers better security, acumen, swiftness and convenience. Consumers can be used ATM machine connected to bank networks. It saves time of both customers and service providers. It reduces labour costs for the bank and upsurge availability of banking in an innocuous and cost effectiveness scheme. In order to enhance the value to the consumers, a magnetic card is given to the customers which is specific to their accounts. Customers can easily access their ATM cards for attaining banking service such as cash withdrawal, balance inquiry, pre-paid mobile recharge, funds transfer, bill payment, request for check book, print out mini statement etc. High reliability of the ATM becomes more significant issues in daily life and necessary for the relation between the banks and the customers. Reliability is a key element in performance evaluation and life testing of the ATM system. Conventional reliability theory deliberates the suppositions of the probability theory and the binary states of a component or sub-systems of the system as operational or failed.

Matutes and Padilla (1994) discussed the encouragements of banks in concern of ATM and derived the implications for ATM compatibility of withdrawal fees, interchange bank fees, entry and depositor switching costs. Davies et al. (1996) discussed how neural networks can bring together psychometric and econometric approaches to the measurements of attitudes and sensitivities; and advantages of usage of neural network. ATM technology perhaps is the most complex networking technology. The

\footnotetext{
*E-mail address: drmrswami@yahoo.com

ISSN: $1791-2377$ @ 2016 Eastern Macedonia and Thrace Institute of Technology. All rights reserved.
}

goal of ATM is to provide a unified networking platform and communication infrastructure, ATM security, as a part of this infrastructure, has to be flexible and compatible with other technology [Liang (1999)]. Howcroft et al. (2002) developed the understanding of consumer attitude towards bank delivery channels and obtained information about the factors which is significant for consumers in encouraging and discouraging the adoption of home based banking. ATM means neither "avoids traveling with money" nor "any time money," but certainly implies both. The inter-bank ATM networks facilitate the use of ATM cards of one bank at the ATM(s) of other banks for basic services like cash withdrawal and balance enquiry [Singh and Komal (2009)]. Adepoju and Alhassan (2010) said that the use of ATM is not only safe but is also convenient. This safety and convenience, unfortunately, has an evil side as well that do not originate from the use of plastic money but rather by the misuse of the same. This evil side is reflected in the form of "ATM frauds" that is a global problem. Ahaiwe (2011) studied the relationship between banks and other service provider in banking sector in the concern of economy and proficient ATM services. ATM organization encourages competition by save time and reduces bank risks. The motivating factors for using the branch ATM services are privacy in carrying out banking transactions, time saving element and the flexibility in use and demotivating factors that prevented respondents from using the branch. Narteh (2013) analysed the five aspects of ATM service quality as reliability of ATM, convenience, responsiveness, ease of use and fulfilment. He founded results provide a guide to bank managers by which improve the efficiency and effectiveness of the ATMs.

Many researchers have done a lot of work related to ATM and ATM network but they have not found the reliability measures of ATM network which have a great importance in the concern of performance of ATM network. 
In the modern scenario, reliability has become the most challenging, demanding theory and can be seen in the literature. Avizienis et al. (2004) discussed the very essential and widespread explanation in the form of basic concepts and taxonomy of dependability and secure computing, and they tried to give the foremost fields and approaches of engineering and sciences, where reliability theory has been applied. Maintaining a high reliability is an essential requirement of ATM network too. In order to increase the efficiency of any network, failed unit is renewed by replacing or repaired. Repairman is also one of the crucial parts of the repairable systems that can affect the economy of the systems directly or indirectly [Manglik and Ram (2013)]. Ram (2013) stated that reliability prediction is the combination of the creation of the proper reliability model together with approximating input parameters for this model, and provide a system level estimate for output reliability parameters.

\section{Mathematical Model details}

\subsection{Assumptions}

The designed model associated with the following assumptions-

i. The ATM system has three possible modes normal, partial failure and total failure.

ii. Failure and repair rates are generally distributed.

iii. Failures are announcing on the basis of literature.

iv. A single repair facility is available to attend the partially or totally failed system.

v. Repairs are perfect i.e. repair facility never does any damage to the units of the network.

vi. The unit recovers its functioning perfectly upon repair.

vii. The system becomes inoperable on the failure of ATM machine or hub of the network.

viii. The repair time of the failed system is arbitrarily distributed.

\subsection{Nomenclature and state description}

Notations associated with this work are shown in Table 1.

Table 1. Notations and states

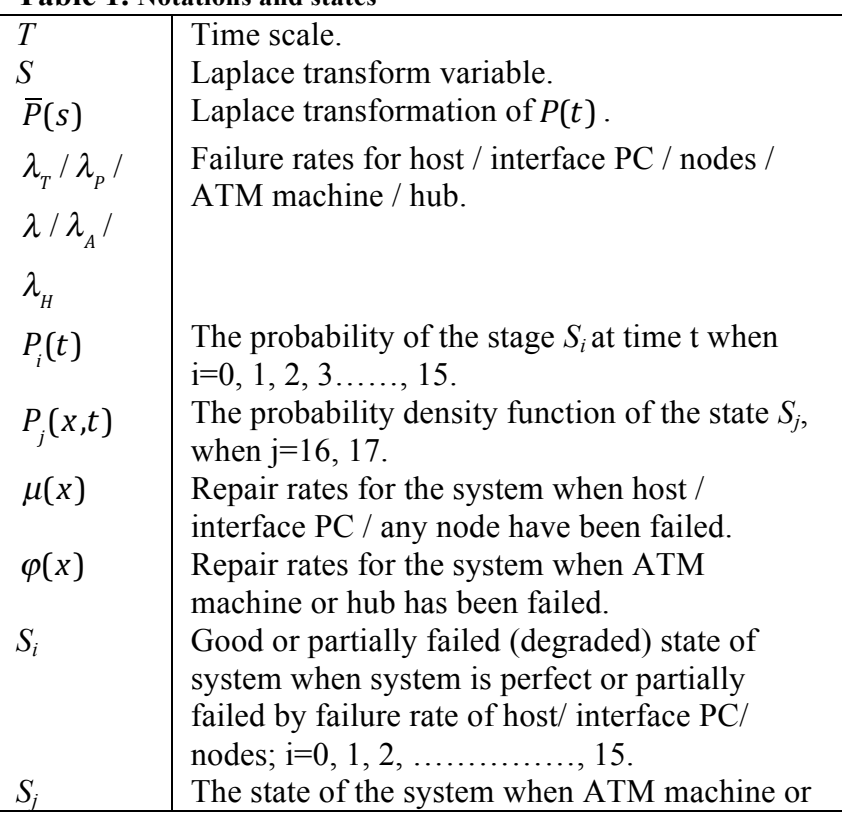

\begin{tabular}{l|l}
\hline & hub is failed; $\mathrm{j}=16,17$. \\
$P_{u p}(t)$ & $\begin{array}{l}\text { Up state system probability at time t or } \\
\text { availability of the ATM system }\end{array}$ \\
$R l(t)$ & The reliability of the ATM system at time t. \\
$E_{p}(t)$ & Expected profit during the interval $[0, \mathrm{t})$. \\
$K_{l}, K_{2}$ & $\begin{array}{l}\text { Revenue and service cost per unit time } \\
\text { respectively. }\end{array}$ \\
\hline
\end{tabular}

\subsection{Problem statement}

This research work investigates about the performance of ATM channel, it is a network in which ATM machine is connected to the host and interface PC with the switch (Hub); nodes for record the transaction of customers. Account is also connected to the host by hub. Configuration diagram of the network is shown in Fig.1(a) and Fig.1(b) demonstrates their state transition diagram. It is assumed that host, interface PC, and nodes are connected in parallel and three nodes are used in the network. This type of ATM network can be easily found in any banking service. The system has general distribution of equipment failures and two repair rates. When hub or ATM machine is failed then system goes to complete failed state otherwise system is partially failed with any other failure of the network. This network can be used for places where the user volume is high. The authors have designed transition state diagram of this network using stochastic modelling which has total 18 states; 1 is good state, 15 partially failed (degraded) states and 2 is complete failed states. When any equipment of the designed system is failed then system is immediately repair and emanate back in good working condition.

\subsection{Formulation and Solution of the Model}

By probabilistic considerations and continuity arguments, we have obtained the following set of differential equations overriding the present mathematical model

$$
\begin{aligned}
& {\left[\frac{\partial}{\partial t}+\lambda_{A}+\lambda_{H}+\lambda_{P}+\lambda_{T}+3 \lambda\right] P_{0}(t)=} \\
& \mu\left(P_{1}(t)+P_{6}(t)+P_{11}(t)\right)+\int_{0}^{\infty} \varphi(x)\left(P_{16}(x, t)+P_{16}(x, t)\right) d x
\end{aligned}
$$

$$
\begin{aligned}
& {\left[\frac{\partial}{\partial t}+\lambda_{T}+\lambda_{H}+\mu\right] P_{i}(t)=\mu P_{i+1}(t)+\lambda_{P} P_{i-1}(t),} \\
& i=1,14 \\
& {\left[\frac{\partial}{\partial t}+3 \lambda+\lambda_{H}+\mu\right] P_{i}(t)=\mu P_{i+1}(t)+\lambda_{T} P_{j}(t) ;} \\
& i=2,6 ; \quad j=1,0
\end{aligned}
$$

$$
\begin{aligned}
& {\left[\frac{\partial}{\partial t}+2 \lambda+\lambda_{H}+\mu\right] P_{i}(t)=\mu P_{i+1}(t)+3 \lambda P_{j}(t) ;} \\
& \quad i=3,7,11 ; \quad j=2,6,0
\end{aligned}
$$$$
\left[\frac{\partial}{\partial t}+\lambda+\lambda_{H}+\mu\right] P_{i}(t)=\mu P_{i+1}(t)+2 \lambda P_{i-1}(t) ;
$$

$$
i=4,8,12
$$


$\left[\frac{\partial}{\partial t}+\lambda_{H}+\mu\right] P_{i}(t)=\alpha P_{i-1}(t)$

$i=5,10,15 ; \quad \alpha=\lambda, \lambda_{P}, \lambda_{T}$

$\left[\frac{\partial}{\partial t}+\lambda_{P}+\lambda_{H}+\mu\right] P_{i}(t)=\mu P_{i+1}(t)+\lambda P_{i-1}(t)$

$i=9,13$
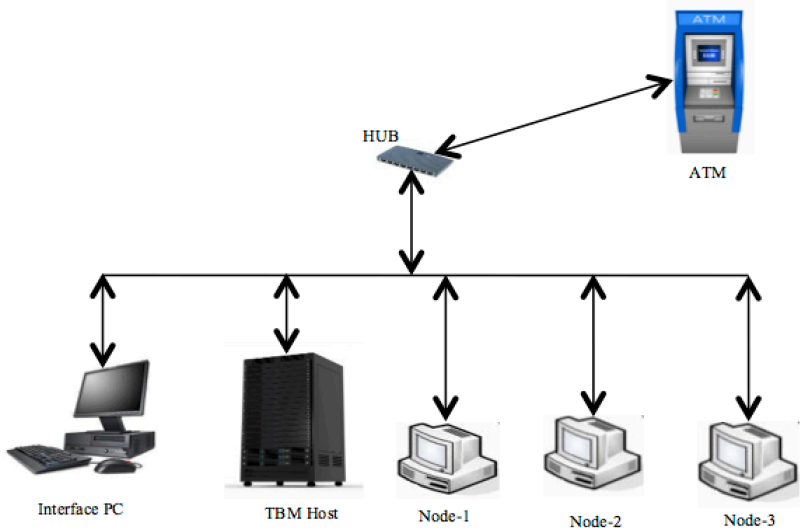

Fig.1(a). Configuration diagram

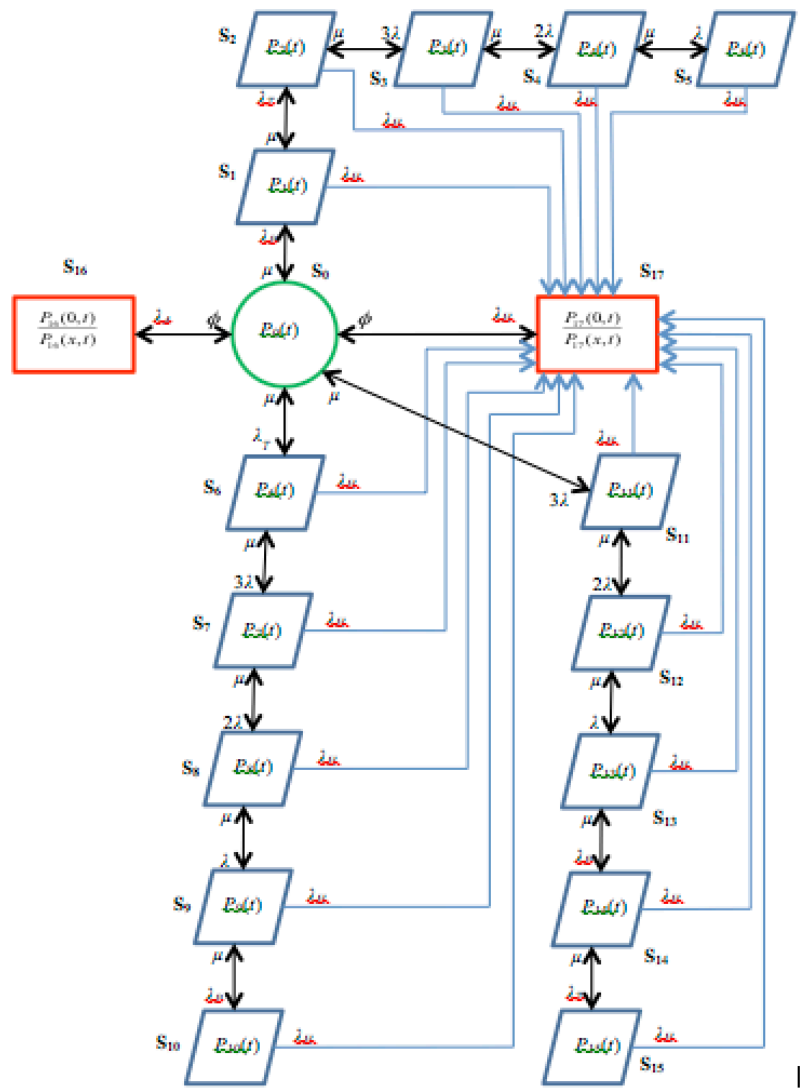

Fig. 1(b). State transition diagram

$\left[\frac{\partial}{\partial t}+\frac{\partial}{\partial x}+\varphi(x)\right] P_{i}(x, t)=0$,

$i=16,17$
Boundary conditions

$$
P_{16}(0, t)=\lambda_{A} P_{0}(t)
$$

$$
P_{17}(0, t)=\lambda_{H} \sum_{i=0}^{15} P_{i}(t)
$$

Initial condition

$P_{i}(0)=1$, when $i=0$ otherwise 0.

Taking Laplace transform of (1) to (10) using (11)

$$
\begin{aligned}
& {\left[s+\lambda_{A}+\lambda_{H}+\lambda_{P}+\lambda_{T}+3 \lambda\right] \bar{P}_{0}(s)=} \\
& 1+\int_{0}^{\infty} \varphi(x)\left(\bar{P}_{16}(x, s)+\bar{P}_{16}(x, s)\right) d x \\
& +\mu\left(\bar{P}_{1}(s)+\bar{P}_{6}(s)+\bar{P}_{11}(s)\right) \\
& {\left[s+\lambda_{T}+\lambda_{H}+\mu\right] \bar{P}_{i}(s)=\mu \bar{P}_{i+1}(s)+\lambda_{P} \bar{P}_{i-1}(s) ; i=1,14}
\end{aligned}
$$

$\left[s+3 \lambda+\lambda_{H}+\mu\right] \bar{P}_{i}(s)=\mu \bar{P}_{i+1}(s)+\lambda_{T} \bar{P}_{j}(s) ;$

$i=2,6 ; \quad j=1,0$

$\left[s+2 \lambda+\lambda_{H}+\mu\right] \bar{P}_{i}(t)=\mu \bar{P}_{i+1}(s)+3 \lambda \bar{P}_{j}(s) ;$

$i=3,7,11 ; \quad j=2,6,0$

$\left[s+\lambda+\lambda_{H}+\mu\right] \bar{P}_{i}(s)=\mu \bar{P}_{i+1}(s)+2 \lambda \bar{P}_{i-1}(s)$;

$$
i=4,8,12
$$

$\left[s+\lambda_{H}+\mu\right] \bar{P}_{i}(s)=\alpha \bar{P}_{i-1}(s)$

$i=5,10,15 ; \quad \alpha=\lambda, \lambda_{P}, \lambda_{T}$

$\left[s+\lambda_{P}+\lambda_{H}+\mu\right] \bar{P}_{i}(s)=\mu \bar{P}_{i+1}(s)+\lambda \bar{P}_{i-1}(s) ;$

$i=9,13$

$\left[s+\frac{\partial}{\partial x}+\varphi(x)\right] \bar{P}_{i}(x, s)=0 ; \quad i=16,17$

$\bar{P}_{16}(0, s)=\lambda_{A} \bar{P}_{0}(s)$

$\bar{P}_{17}(0, s)=\lambda_{H} \sum_{i=0}^{15} \bar{P}_{i}(s)$

After solving Equation (12) to (21), we get the state transition probabilities as

(8) $\quad \bar{P}_{0}(s)=\frac{1}{D(s)}$ 


$$
\begin{aligned}
& \bar{P}_{i}(s)=\frac{A_{j-1} \xi}{A_{j}} \bar{P}_{0}(s) \text {; } \\
& i=1,6,11 ; \quad j=12,8,4 ; \quad \xi=\lambda_{P}, \lambda_{T}, 3 \lambda \\
& \bar{P}_{i}(s)=\frac{A_{j-2} \xi}{A_{j}} \bar{P}_{0}(s) ; \\
& i=2,7,12 ; j=12,8,4 ; \quad \xi=\lambda_{P} \lambda_{T}, 3 \lambda \lambda_{T}, 6 \lambda^{2} \\
& \bar{P}_{i}(s)=\frac{A_{j-3} \xi}{A_{j}} \bar{P}_{0}(s) \text {; } \\
& i=3,8,13 ; \quad j=12,8,4 ; \quad \xi=3 \lambda_{P} \lambda_{T} \lambda, 6 \lambda_{T} \lambda^{2}, 6 \lambda^{3} \\
& \bar{P}_{i}(s)=\frac{6 \xi\left(s+H_{7}\right)}{A_{j}} \bar{P}_{0}(s) ; \\
& i=4,9,14 ; j=12,8,4 ; \quad \xi=\lambda_{P} \lambda_{T} \lambda^{2}, \lambda_{T} \lambda^{3}, \lambda_{P} \lambda^{3} \\
& \bar{P}_{i}(s)=\frac{6 \lambda_{P} \lambda_{T} \lambda^{3}}{A_{j}} \bar{P}_{0}(s) ; \quad i=5,10,15 ; \quad j=12,8,4 \\
& \bar{P}_{i}(s)=\alpha \beta \xi ; \quad \alpha=\lambda_{A}, \lambda_{H} ; \quad \beta=\left(\frac{1-\bar{S}_{\varphi}(s)}{s}\right) ; \\
& \xi=\bar{P}_{0}(s), \sum_{i=0}^{15} \bar{P}_{i}(s)
\end{aligned}
$$

Where

$$
\begin{aligned}
& D(s)=\left(s+H_{1}\right)-\mu\left\{\frac{A_{11} \lambda_{P}}{A_{12}}+\frac{A_{7} \lambda_{T}}{A_{8}}+\frac{3 A_{3} \lambda}{A_{4}}\right\}-\bar{S}_{\varphi}(s)\left(\lambda_{A}+\lambda_{A} B\right) \\
& H_{1}=\lambda_{P}+\lambda_{T}+\lambda_{H}+\lambda_{A}+3 \lambda, \quad H_{2}=\lambda_{T}+\lambda_{H}+\mu, \\
& H_{3}=\lambda_{P}+\lambda_{H}+\mu, \quad H_{4}=\lambda_{H}+3 \lambda+\mu, \quad H_{5}=\lambda_{H}+2 \lambda+\mu, \\
& H_{6}=\lambda_{H}+\lambda+\mu, \\
& H_{7}=\lambda_{H}+\mu \\
& A_{1}=\left(s+H_{2}\right)\left(s+H_{7}\right)-\mu \lambda_{T} ; \quad A_{2}=\left(s+H_{3}\right) A_{1}-\mu \lambda_{P}\left(s+H_{7}\right) ; \\
& A_{3}=\left(s+H_{6}\right) A_{2}-\mu \lambda A_{1} ; A_{4}=\left(s+H_{5}\right) A_{3}-2 \mu \lambda A_{2} ; \\
& A_{5}=\left(s+H_{3}\right)\left(s+H_{7}\right)-\mu \lambda_{P} ; \quad A_{6}=\left(s+H_{6}\right) A_{5}-\mu \lambda\left(s+H_{7}\right) ; \\
& A_{7}=\left(s+H_{5}\right) A_{6}-2 \mu \lambda A_{5} ; A_{8}=\left(s+H_{4}\right) A_{7}-3 \mu \lambda A_{6} ; \\
& A_{9}=\left(s+H_{6}\right)\left(s+H_{7}\right)-\mu \lambda ; \quad A_{10}=\left(s+H_{5}\right) A_{9}-2 \mu \lambda\left(s+H_{7}\right) ; \\
& A_{11}=\left(s+H_{4}\right) A_{10}-3 \mu \lambda A_{9} ; A_{12}=\left(s+H_{2}\right) A_{11}-\mu \lambda_{T} A_{10}
\end{aligned}
$$

Laplace transformation of probability of the state when the system is in up-state (degraded or good state)

$$
\begin{aligned}
& \bar{P}_{u p}(s)=\sum_{i=0}^{15} \bar{P}_{i}(s) \\
& =\left\{1+\frac{A_{11} \lambda_{P}+A_{10} \lambda_{P} \lambda_{T}+3 A_{9} \lambda_{P} \lambda_{T} \lambda+6 \lambda_{P} \lambda_{T} \lambda^{2}\left(s+H_{7}\right)+6 \lambda_{P} \lambda_{T} \lambda^{3}}{A_{12}}\right.
\end{aligned}
$$$$
+\frac{A_{7} \lambda_{T}+3 A_{6} \lambda \lambda_{T}+6 A_{5} \lambda_{T} \lambda^{2}+6 \lambda_{T} \lambda^{3}\left(s+H_{7}\right)+6 \lambda_{P} \lambda_{T} \lambda^{3}}{A_{8}}
$$$$
\left.+\frac{3 A_{3} \lambda+6 A_{2} \lambda^{2}+6 A_{1} \lambda^{3}+6 \lambda_{P} \lambda_{T} \lambda^{3}+6 \lambda_{P} \lambda^{3}\left(s+H_{7}\right)}{A_{4}}\right\} \bar{P}_{0}(s)
$$

Laplace transformation of probability of the state when the system is in down-state (failed state)

$$
\begin{aligned}
& \bar{P}_{\text {down }}(s)=\bar{P}_{16}(s)+\bar{P}_{17}(s) \\
& =\left(\frac{1-\bar{S}_{\varphi}(s)}{s}\right)\left[\lambda_{A}+\lambda_{H}\left\{1+\frac{A_{11} \lambda_{P}+A_{10} \lambda_{P} \lambda_{T}+3 A_{9} \lambda_{P} \lambda_{T} \lambda+6 \lambda_{P} \lambda_{T} \lambda^{2}\left(s+H_{7}\right)+6 \lambda_{P} \lambda_{T} \lambda^{3}}{A_{12}}\right.\right. \\
& +\frac{A_{7} \lambda_{T}+3 A_{6} \lambda \lambda_{T}+6 A_{5} \lambda_{T} \lambda^{2}+6 \lambda_{T} \lambda^{3}\left(s+H_{7}\right)+6 \lambda_{P} \lambda_{T} \lambda^{3}}{A_{8}} \\
& \left.+\frac{3 A_{3} \lambda+6 A_{2} \lambda^{2}+6 A_{1} \lambda^{3}+6 \lambda_{P} \lambda_{T} \lambda^{3}+6 \lambda_{P} \lambda^{3}\left(s+H_{7}\right)}{A_{4}}\right\} \bar{P}_{0}(s)
\end{aligned}
$$

\section{Particular cases and Numerical computations}

\subsection{Availability Analysis}

Availability may be progressed by an elaborate plan on focusing on increasing testability and maintainability and not on reliability. The value of availability depends on the system structure as well as on the component availability. This value decreases as the component ages increases, i.e. their serving times are influenced by their interactions with each other's [Samrout et al. (2005)].

\section{(i) When the ATM system is in comprehensive state}

Consider that initially, the ATM system works properly. We assume that $100 \%$ repair facility is available in the system, hence taking the repair rates as $\varphi(x)=1, \mu(x)=1$ and value of different failure rates as $\lambda_{T}=0.15, \lambda_{P}=0.05$, $\lambda=0.2, \lambda_{A}=0.2, \lambda_{H}=0.1$ in Equation (29). We obtain the availability in terms of time after taking the inverse Laplace transformation.

\section{(ii) When no failure in the host of the ATM system}

Suppose that the host failure rate is zero; and setting other failure and repair rates as $\lambda_{T}=0, \lambda_{P}=0.05, \lambda=0.2$, $\lambda_{A}=0.2, \lambda_{H}=0.1, \varphi(x)=1, \mu(x)=1$ in Equation (29) and find the inverse Laplace transformation. One can obtain availability of the ATM system in terms of time. 
(iii) When no failure in the interface PC of the ATM system

Consider that the interface PC failure rate is zero and other different failure rates and repair rates as $\lambda_{T}=0.15, \lambda_{P}=0$, $\lambda=0.2, \lambda_{A}=0.2, \lambda_{H}=0.1, \varphi(x)=1, \mu(x)=1$. Substituting all these values in Equation (29) and taking the inverse Laplace transformation, one can determine the availability of the ATM system in terms of time.

\section{(iv) When no failure in nodes of the ATM network}

When nodes in the ATM network are not failed then failure rate of node is zero. Taking the value of different failure rates and repair rates as $\lambda_{T}=0.15, \lambda_{P}=0.05, \lambda=0$, $\lambda_{A}=0.2, \lambda_{H}=0.1, \varphi(x)=1, \mu(x)=1$. Putting all these values in Equation (29), we may get availability in terms of time after taking the inverse Laplace transformation.

\section{(v) When no failure in ATM machine}

When ATM machine is in perfect working condition, fixing the value of different failure rates and repair rates as $\lambda_{T}=0.15, \lambda_{P}=0.05, \lambda=0.2, \lambda_{A}=0, \lambda_{H}=0.1, \varphi(x)=1$,

$\mu(x)=1$ and substituting all these values in Equation (29), we obtain availability in terms of time after taking the inverse Laplace transformation.

\section{(vi) When no failure in hub of the ATM network}

Contemplate that hub in the network is not failed, taking the value of different failure rates and repair rates as $\lambda_{T}=0.15$, $\lambda_{P}=0.05, \lambda=0.2, \lambda_{A}=0.2, \lambda_{H}=0, \varphi(x)=1, \mu(x)=1$ and substitute all these values in Equation (29), we obtain the availability in terms of time after finding the inverse Laplace transformation.

Now varying the time unit $\mathrm{t}$ from 0 to 20 in the inverse Laplace transformation of above cases, one can get the availability of the ATM system is shown in Table 2 and represented graphically in Fig.2.

Table 2. Availability as function of time

\begin{tabular}{c|c|c|c|c|c|c}
\hline \multirow{2}{*}{$\begin{array}{c}\text { Time } \\
\text { (t) }\end{array}$} & \multicolumn{7}{|c}{ Availability $\boldsymbol{P}_{\text {up }}(\boldsymbol{t})$} \\
\cline { 2 - 7 } ) & Case (i) & $\begin{array}{c}\text { Case } \\
\text { (ii) }\end{array}$ & $\begin{array}{c}\text { Case } \\
\text { (ii) }\end{array}$ & $\begin{array}{c}\text { Case } \\
\text { (iv) }\end{array}$ & $\begin{array}{c}\text { Case } \\
\text { (v) }\end{array}$ & $\begin{array}{c}\text { Case } \\
\text { (vi) }\end{array}$ \\
\hline 0 & 1.00000 & 1.00000 & 1.00000 & 1.00000 & 1.00000 & 1.00000 \\
1 & 0.86030 & 0.85587 & 0.85892 & 0.84022 & 0.93935 & 0.91927 \\
2 & 0.83591 & 0.82883 & 0.83393 & 0.80134 & 0.91916 & 0.90683 \\
3 & 0.83229 & 0.82430 & 0.83027 & 0.79167 & 0.91244 & 0.90917 \\
4 & 0.83246 & 0.82410 & 0.83048 & 0.78922 & 0.91021 & 0.91228 \\
5 & 0.83307 & 0.82454 & 0.83113 & 0.78859 & 0.90946 & 0.91447 \\
6 & 0.83354 & 0.82491 & 0.83161 & 0.78842 & 0.90921 & 0.91583 \\
7 & 0.83383 & 0.82514 & 0.83190 & 0.78838 & 0.90913 & 0.91666 \\
8 & 0.83400 & 0.82528 & 0.83207 & 0.78837 & 0.90910 & 0.91716 \\
9 & 0.83410 & 0.82535 & 0.83217 & 0.78837 & 0.90909 & 0.91746 \\
10 & 0.83416 & 0.82539 & 0.83222 & 0.78837 & 0.90909 & 0.91764 \\
11 & 0.83419 & 0.82541 & 0.83225 & 0.78837 & 0.90909 & 0.91776 \\
12 & 0.83421 & 0.82542 & 0.83227 & 0.78837 & 0.90909 & 0.91783 \\
13 & 0.83422 & 0.82542 & 0.83228 & 0.78837 & 0.90909 & 0.91788 \\
14 & 0.83422 & 0.82543 & 0.83229 & 0.78837 & 0.90909 & 0.91791 \\
15 & 0.83423 & 0.82543 & 0.83229 & 0.78837 & 0.90909 & 0.91794 \\
16 & 0.83423 & 0.82543 & 0.83229 & 0.78837 & 0.90909 & 0.91795 \\
17 & 0.83423 & 0.82543 & 0.83229 & 0.78837 & 0.90909 & 0.91795 \\
18 & 0.83423 & 0.82543 & 0.83229 & 0.78837 & 0.90909 & 0.91796 \\
19 & 0.83423 & 0.82543 & 0.83229 & 0.78837 & 0.90909 & 0.91797 \\
20 & 0.83423 & 0.82543 & 0.83229 & 0.78837 & 0.90909 & 0.91797 \\
\hline
\end{tabular}

\subsection{Reliability Analysis}

Reliability of a network is an essential aspect in the designing of the network because reliability investigates the probability that the network will successfully meet their design requirements. If any key equipment of the network is damaged, the system will break down [Zhang and Yang (2009)]. For procurement the reliability of the system assume that repair facility is not available i.e. taking all the repair rates equal to zero in Equation (29).

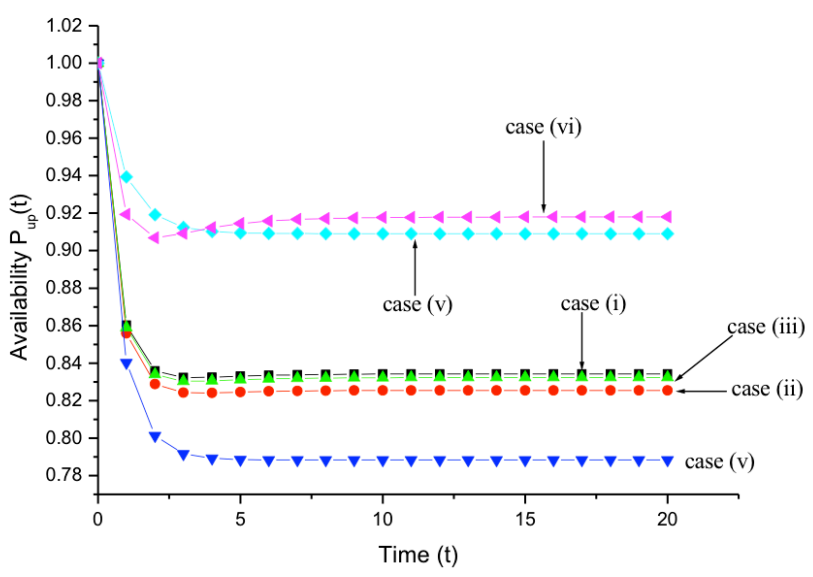

Fig. 2. Availability v/s Time

(i) When the ATM system is in comprehensive state

Consider that initially, the system work properly. For this taking the value of different failure rates as $\lambda_{T}=0.15$, $\lambda_{P}=0.05, \lambda=0.2, \lambda_{A}=0.2, \quad \lambda_{H}=0.1$ in Equation (29). We obtain the reliability in terms of time after taking the inverse Laplace transformation.

\section{(ii) When no failure in the host of the ATM system}

Taking the host failure rate is zero; and other failure as $\lambda_{T}=0, \lambda_{P}=0.05, \lambda=0.2, \lambda_{A}=0.2, \lambda_{H}=0.1$ in Equation (29), we may get the reliability as a function of time after determine the inverse Laplace transformation.

\section{(iii) When no failure in the interface PC of the ATM system}

Suppose that the interface PC failure rate is zero and other different failure rates as $\lambda_{T}=0.15, \lambda_{P}=0, \lambda=0.2, \lambda_{A}=0.2$, $\lambda_{H}=0.1$. Substituting all these values in Equation (29) and taking the inverse Laplace transformation, one can obtain the reliability as a function of time.

\section{(iv) When no failure in nodes of the ATM network}

Consider that any node connected in the network is not failed so its failure rate is zero. Taking the value of different failure rates as $\lambda_{T}=0.15, \lambda_{P}=0.05, \lambda=0, \lambda_{A}=0.2$, $\lambda_{H}=0.1$. Putting all these values in Equation (29) determine the inverse Laplace transformation. One can determine the reliability in terms of time.

\section{(v) When no failure in ATM machine}

Contemplate that ATM machine connected in the network is not failed, setting the value of different failure rates as $\lambda_{T}=0.15, \lambda_{P}=0.05, \lambda=0.2, \lambda_{A}=0, \lambda_{H}=0.1$. Substituting all these values in Equation (29), one can determine the reliability as a function of time after taking the inverse Laplace transformation. 
(vi) When no failure in hub of the ATM network

Contemplate that hub in the network is not failed, fixing the value of different failure rates as $\lambda_{T}=0.15, \lambda_{P}=0.05$,

$\lambda=0.2, \lambda_{A}=0.2, \lambda_{H}=0$. Positioning all these values in Equation (29), one can calculate reliability in terms of time after taking the inverse Laplace transformation.

Now varying the time unit $t$ from 0 to 20 in the inverse Laplace transformation of above cases, one can get the reliability of the ATM network is shown in Table 3 and correspondingly revealed in Fig.3 respectively.

Table 3. Reliability as function of time

\begin{tabular}{c|c|c|c|c|c|c}
\hline \multirow{2}{*}{$\begin{array}{c}\text { Time } \\
\text { (t) }\end{array}$} & \multicolumn{7}{|c|}{ Reliability $\boldsymbol{R}(\boldsymbol{t}$ ) } \\
\cline { 2 - 7 } & case (i) & case (ii) & $\begin{array}{c}\text { case } \\
\text { (iii) }\end{array}$ & $\begin{array}{c}\text { case } \\
\text { (iv) }\end{array}$ & case (v) & $\begin{array}{c}\text { case } \\
\text { (vi) }\end{array}$ \\
\hline 0 & 1.00000 & 1.00000 & 1.00000 & 1.00000 & 1.00000 & 1.00000 \\
1 & 0.79044 & 0.78293 & 0.78802 & 0.75568 & 0.90484 & 0.87357 \\
2 & 0.67714 & 0.66128 & 0.67215 & 0.59330 & 0.81873 & 0.82707 \\
3 & 0.60003 & 0.58012 & 0.59388 & 0.48197 & 0.74082 & 0.80996 \\
4 & 0.53871 & 0.51786 & 0.53236 & 0.40283 & 0.67032 & 0.80366 \\
5 & 0.48604 & 0.46585 & 0.47994 & 0.34431 & 0.60653 & 0.80135 \\
6 & 0.43932 & 0.42047 & 0.43366 & 0.29930 & 0.54881 & 0.80049 \\
7 & 0.39736 & 0.38005 & 0.39218 & 0.26339 & 0.49658 & 0.80018 \\
8 & 0.35949 & 0.34372 & 0.35478 & 0.23382 & 0.44933 & 0.80007 \\
9 & 0.32526 & 0.31095 & 0.32099 & 0.20884 & 0.40657 & 0.80002 \\
10 & 0.29431 & 0.28134 & 0.29044 & 0.18731 & 0.36788 & 0.80001 \\
11 & 0.26630 & 0.25455 & 0.26279 & 0.16848 & 0.33287 & 0.80000 \\
12 & 0.24095 & 0.23033 & 0.23778 & 0.15184 & 0.30119 & 0.80000 \\
13 & 0.21802 & 0.20841 & 0.21516 & 0.13702 & 0.27253 & 0.80000 \\
14 & 0.19727 & 0.18857 & 0.19468 & 0.12375 & 0.24660 & 0.80000 \\
15 & 0.17850 & 0.17063 & 0.17615 & 0.11184 & 0.22313 & 0.80000 \\
16 & 0.16152 & 0.15439 & 0.15939 & 0.10111 & 0.20190 & 0.80000 \\
17 & 0.14615 & 0.13970 & 0.14422 & 0.09144 & 0.18268 & 0.80000 \\
18 & 0.13224 & 0.12640 & 0.13050 & 0.08271 & 0.16530 & 0.80000 \\
19 & 0.11965 & 0.11438 & 0.11808 & 0.07482 & 0.14957 & 0.80000 \\
20 & 0.10827 & 0.10349 & 0.10684 & 0.06769 & 0.13533 & 0.80000 \\
\hline
\end{tabular}

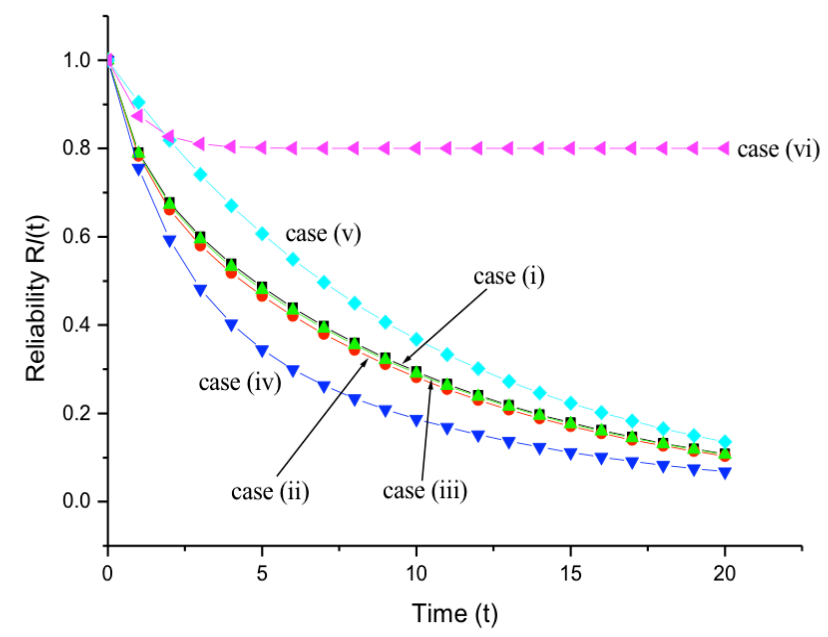

Fig. 3. Reliability v/s Time

\subsection{Mean time to failure (MTTF)}

Mean time to failure (MTTF) is the forecasted elapsed time between inherent failures of a system during operation. It can be determined as the average time between failures of a network. Considering all repair rates to be zero in (29), one can obtain the MTTF as

$$
\begin{aligned}
& M T T F=\lim _{s \rightarrow 0} \bar{P}_{u p}(s) \\
& =\frac{\lambda_{P}+3 \lambda+\lambda_{H}+\lambda_{T}}{\left(\lambda_{P}+3 \lambda+\lambda_{H}+\lambda_{T}+\lambda_{A}\right) \lambda_{H}}
\end{aligned}
$$

Now varying input parameters one by one at $0.1,0.2, \ldots \ldots \ldots \ldots \ldots, 0.9$ respectively and setting the failure rate as $\lambda_{T}=0.15, \lambda_{P}=0.05, \lambda=0.2, \lambda_{A}=0.2, \lambda_{H}=0.1$ in (31), we can get the variation of MTTF with respect to failure rates that signify in Table 4 and graphical representation shown in Fig.4 as

Table 4. MTTF as function of failure rates

\begin{tabular}{c|c|c|c|c|c}
\hline \multirow{2}{*}{$\begin{array}{c}\text { Variation in } \\
\lambda_{T}, \lambda_{P}, \lambda, \lambda_{A},\end{array}$} & \multicolumn{5}{|c}{ MTTF with respect to failure rates } \\
\cline { 2 - 6 }$\lambda_{H}$ & $\lambda_{T}$ & $\lambda_{P}$ & $\lambda$ & $\lambda_{A}$ & $\lambda_{H}$ \\
\hline 0.1 & & & & & \\
0.2 & 8.09524 & 8.26087 & 7.50000 & 9.00000 & 8.18182 \\
0.3 & 8.26087 & 8.40000 & 8.18182 & 8.18182 & 4.16667 \\
0.4 & 8.40000 & 8.51852 & 8.57143 & 7.50000 & 2.82051 \\
0.5 & 8.51852 & 8.62069 & 8.82353 & 6.92308 & 2.14286 \\
0.6 & 8.62069 & 8.70968 & 9.00000 & 6.42857 & 1.73333 \\
0.7 & 8.70968 & 8.78788 & 9.13043 & 6.00000 & 1.45833 \\
0.8 & 8.78788 & 8.85714 & 9.23077 & 5.62500 & 1.26050 \\
0.9 & 8.85714 & 8.91892 & 9.31034 & 5.29412 & 1.11111 \\
& 8.91892 & 8.97436 & 9.37500 & 5.00000 & 0.99415 \\
\hline
\end{tabular}

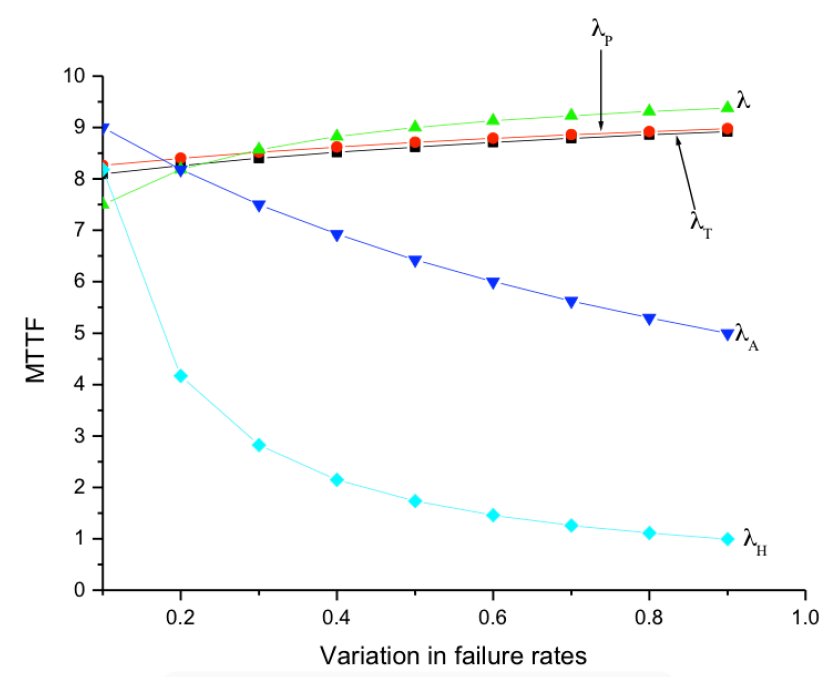

Fig. 4. MTTF as function of failure rates

\subsection{Sensitivity}

Sensitivity of a measure is described as the partial derivative of the measure with respect to their input factors. Sensitivity analysis, also called importance analysis [Henley and Kumamoto (1992), Andrews and Moss (1993)], help detect which parameter contribute most to system performance and thus would be good ones for elevate. Sensitivity of a feature is defined as the partial derivative of the function with respect to their input factors. Here these input factors are failure rates of the designed ATM system.

\subsubsection{Sensitivity of reliability}

Reliability sensitivity can be obtained by differentiating partially (29) after putting repair rates zero, with respect to their input parameters. Setting the values of input parameters as in comprehensive case of reliability analysis, one can obtain Table 5 and their graphical representation as shown in Fig.5.

\subsubsection{Sensitivity of MTTF}

MTTF sensitivity can be predicted by partial differentiation of (31) with respect to the input parameters and then setting the values of input parameters as in partial derivatives of MTTF, one may get Table 6 and graphically demonstrated by Fig. 6 
Table 5. Reliability sensitivity as a function of time

\begin{tabular}{c|l|l|l|l|l}
\hline \multirow{2}{*}{ Time (t) } & \multicolumn{5}{|c}{ Reliability Sensitivity } \\
\cline { 2 - 6 } & & & & & \\
\hline 0 & 0.00000 & 0.00000 & 0.00000 & 0.00000 & 0.00000 \\
1 & 0.04782 & 0.04782 & 0.14346 & -0.52415 & -0.79044 \\
2 & 0.09726 & 0.09726 & 0.29179 & -0.61066 & -1.35429 \\
3 & 0.11866 & 0.11866 & 0.35597 & -0.58528 & -1.80009 \\
4 & 0.12179 & 0.12179 & 0.36536 & -0.53626 & -2.15485 \\
5 & 0.11640 & 0.11640 & 0.34921 & -0.48604 & -2.43021 \\
6 & 0.10786 & 0.10786 & 0.32357 & -0.43959 & -2.63593 \\
7 & 0.09859 & 0.09859 & 0.29578 & -0.39754 & -2.78151 \\
8 & 0.08959 & 0.08959 & 0.26878 & -0.35958 & -2.87595 \\
9 & 0.08121 & 0.08121 & 0.24364 & -0.32530 & -2.92739 \\
10 & 0.07354 & 0.07354 & 0.22062 & -0.29432 & -2.94307 \\
11 & 0.06656 & 0.06656 & 0.19968 & -0.26630 & -2.92928 \\
12 & 0.06023 & 0.06023 & 0.18070 & -0.24096 & -2.89147 \\
13 & 0.05450 & 0.05450 & 0.16351 & -0.21803 & -2.83433 \\
14 & 0.04932 & 0.04932 & 0.14796 & -0.19728 & -2.76189 \\
15 & 0.04462 & 0.04462 & 0.13388 & -0.17850 & -2.67756 \\
16 & 0.04038 & 0.04038 & 0.12114 & -0.16152 & -2.58427 \\
17 & 0.03654 & 0.03654 & 0.10961 & -0.14615 & -2.48449 \\
18 & 0.03306 & 0.03306 & 0.09918 & -0.13224 & -2.38030 \\
19 & 0.02991 & 0.02991 & 0.08974 & -0.11965 & -2.27344 \\
20 & 0.02707 & 0.02707 & 0.08120 & -0.10827 & -2.16536 \\
\hline
\end{tabular}

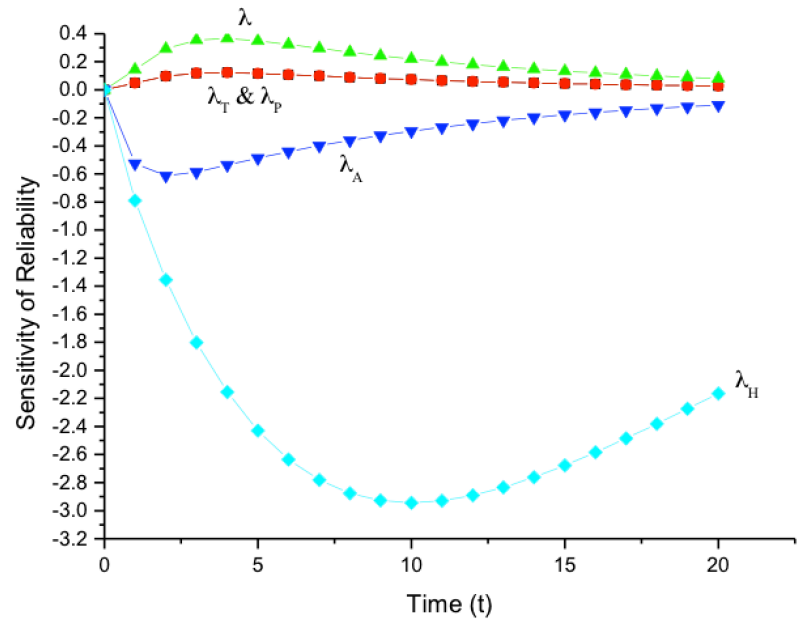

Fig. 5. Reliability sensitivity V/s Time

Table 6. MTTF sensitivity as a function of input parameters

\begin{tabular}{c|c|c|c|c|c}
\hline $\begin{array}{c}\text { Variatio } \\
\mathbf{n} \text { in } \\
\lambda_{T},\end{array}$ & \multicolumn{5}{|c}{ MTTF Sensitivity } \\
\cline { 3 - 6 }$\lambda_{P}$, & & & & & \\
$\lambda$, & $\frac{\partial M T T F}{\partial \lambda_{T}}$ & $\frac{\partial M T T F}{\partial \lambda_{P}}$ & $\frac{\partial M T T F}{\partial \lambda}$ & $\frac{\partial M T T F}{\partial \lambda_{A}}$ & $\frac{\partial M T T F}{\partial \lambda_{H}}$ \\
$\lambda_{A}$, & & & & & \\
$\lambda_{H}$ & & & & & \\
\hline 0.1 & 1.81406 & 1.51229 & 9.37500 & -9.00000 & -80.16529 \\
0.2 & 1.51229 & 1.28000 & 4.95868 & -7.43802 & -20.13889 \\
0.3 & 1.28000 & 1.09739 & 3.06122 & -6.25000 & -9.00723 \\
0.4 & 1.09739 & 0.95125 & 2.07612 & -5.32544 & -5.10204 \\
0.5 & 0.95125 & 0.83247 & 1.50000 & -4.59184 & -3.28889 \\
0.6 & 0.83247 & 0.73462 & 1.13421 & -4.00000 & -2.30035 \\
0.7 & 0.73462 & 0.65306 & 0.88757 & -3.51562 & -1.70186 \\
0.8 & 0.65306 & 0.58437 & 0.71344 & -3.1419 & -1.31173 \\
0.9 & 0.58437 & 0.52597 & 0.58594 & -2.777778 & -1.04305 \\
\hline
\end{tabular}

\section{Results and Discussion}

In this work, authors have sought the performance of ATM network with the help of analysing the availability, reliability, mean time to failure and sensitivity of the designed ATM network. In this section, authors are discussing the above findings.
From Table 2 and Fig.2, one has an idea about the availability of the ATM system. By the graph of availability, it is clear that availability of the designed ATM network decreases fastly as time increases in each case and after a long run it becomes constant at some value. Except in case of no failure in ATM machine or nodes, availability of the system increases slightly within a short period of time and then becomes constant. When nodes are not failed then network has lowest availability and in case of no failure of hub, network has highest availability.

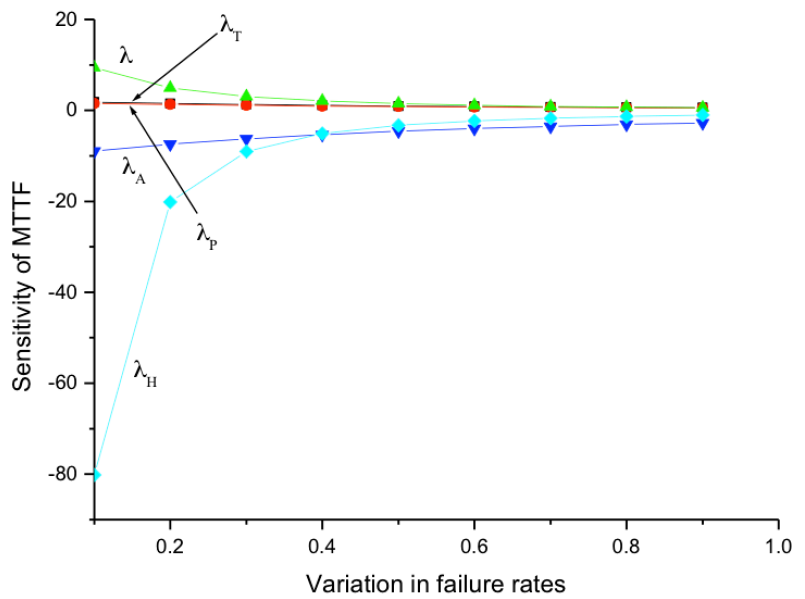

Fig. 6. MTTF sensitivity v/s Failure rates

Table 3 and Fig. 3 gives us an idea about reliability of the ATM network. Critical examination of Fig. 3 shows that how reliable this network? From the graph of reliability, one can see that reliability of suggested network decreases smoothly as time passes and establish at some value after a long run in each situation. It is surprisingly that when hub connected in the network is in perfect working condition then the network is highly reliable. In this situation, it is seen that reliability is less decreases as compare to other situations. In case of perfectly functioning nodes, the network is less reliable. When ATM network is in comprehensive state or when host/interface PC is not failed, the network accomplishes approximately same reliability.

Fig. 4 yields the mean time to failure of ATM network. Generally, MTTF of any system decreases as failure rate increases. In the case of ATM network, MTTF leads decrement with the increment of failure rate of ATM machine or hub of the network. ATM machine and hub is the most significant unit of the channel. It is interesting point that MTTF of ATM network increases when failure rate of host/interface $\mathrm{PC} /$ nodes increases, those play backstage role in this network. These units of ATM channel are used only by service providers in bank. In case of increasing the failure rate of nodes MTTF is highly increase while in case of increasing failure rate of hub MTTF is highly decreases.

Fig. 5 shows the trend of reliability sensitivity. It reveals that the sensitivity of reliability decreases in case of no failure in ATM machine or hub and increases in case of no failure in nodes/ interface $\mathrm{PC} /$ host. Vital examination of the graph shows that when no failure in host and interface PC, the reliability system coincide with each other. One can see that reliability of the network is most affected by the hub failure. By controlling the failure rate of hub, one can improve the reliability of the designed ATM network. The sensitivity of MTTF with respect to their input parameters is 
exposed in Fig.6. From the graph of MTTF sensitivity, one can see that the MTTF sensitivity increases with respect to increasing failure rate of hub and ATM machine and decreases in case of increasing failure rate of host / interface PC / nodes. Sensitivity of MTTF is increases rapidly as failure rate of hub increases. As failure rate of nodes / interface PC / host increases, after some increment sensitivity of MTTF coincide with each other. It concluded that by controlling the failure rate of hub, one can improve the reliability of the designed network and can reduce the MTTF of the network.

\section{Conclusion}

The present study has attempted the comprehensive overview of the performance of an ATM network with the help of availability, reliability, MTTF, and sensitivity of reliability and MTTF of the designed ATM network. Performance is very important for any network or system. Authors concluded that by controlling the failure rate of ATM machine and hub connected in the network, one can improve the reliability of the designed network and increase the MTTF of the network. Hence, this study is very useful for reliability engineers and designers because with the help of this study they can achieve better performance of the ATM network. In future, there is huge opportunity for growth of ATM network in banking sector. More research is required to investigate whether or not a pictorial design could provide appropriate clues for the user; about the security and economy of banking sector.

\section{References}

1. Adepoju, A. S., \& Alhassan, M. E. (2010). Challenges of Automated Teller Machine (ATM) Usage and Fraud Occurrences in Nigeria-A Case Study of Selected Banks in Minna Metropolis. Journal of Internet Banking \& Commerce, 15(2), 1-10.

2. Ahaiwe, J. (2011). The effect of automated teller machines on banks' services in Nigeria. International Journal of Development and Management Review, 6(1).

3. Andrews, J. D., and Moss, T. R. (1993). Reliability and risk assessment. Longman Scientific and Technical.

4. Avizienis, A., Laprie, J. C., Randell, B., \& Landwehr, C. (2004). Basic concepts and taxonomy of dependable and secure computing. IEEE Transactions on Dependable and Secure Computing, 1(1), 11-33.

5. Davies, F., Moutinho, L., \& Curry, B. (1996). ATM user attitudes: a neural network analysis. Marketing Intelligence \& Planning, 14(2), 26-32.

6. Henley, E. J. and Kumamoto H. (1992). Probabilistic Risk Assessment: IEEE Press.

7. Howcroft, B., Hamilton, R., \& Hewer, P. (2002). Consumer attitude and the usage and adoption of home-based banking in the United Kingdom. International Journal of Bank Marketing, 20(3), 111121.

8. Liang, D. (1999). A survey on ATM security. Ohio State University Student Report.
9. Manglik, M., \& Ram, M. (2013). Reliability analysis of a two unit cold standby system using Markov process. Journal of Reliability and Statistical Studies, 6(2), 65-80.

10. Matutes, C., \& Padilla, A. J. (1994). Shared ATM networks and banking competition. European Economic Review, 38(5), 11131138.

11. Narteh, B. (2013). Service quality in automated teller machines: an empirical investigation. Managing Service Quality, 23(1), 62-89.

12. Ram, M. (2013). On system reliability approaches: a brief survey. International Journal of System Assurance Engineering and Management, 4(2), 101-117.

13. Samrout, M., Yalaoui, F., Châtelet, E., and Chebbo, N. (2005). New methods to minimize the preventive maintenance cost of seriesparallel systems using ant colony optimization. Reliability Engineering and System Safety, 89(3), 346-354.

14. Singh, S. K., \& Komal, M. (2009). Impact of ATM on customer satisfaction (a comparative study of SBI, ICICI, \& HDFC bank). Business Intelligence Journal, 2(2), 276-287.

15. Zhang, Y. M., and Yang, Z. (2009). Reliability-based sensitivity analysis of vehicle components with non-normal distribution parameters. International Journal of Automotive Technology, 10(2), 181-194. 